\title{
Looking at Hydra cells one at a time
}

\author{
Single-cell sequencing of Hydra results in a resource for studying differentiation trajectories, regeneration and \\ nervous system development.
}

$\mathrm{H}$ ow stem cells differentiate, and how their progeny populate a developing or regenerating tissue or organism, is an active area of research in developmental biology. Even though Hydra vulgaris is not as widely studied as, for example, Drosophila or the mouse, this organism is particularly suitable for addressing such questions. "Hydra has this really interesting feature where it renews itself all of the time," says Celina Juliano from the University of California, Davis, making it easy to capture differentiation processes.

Juliano and her collaborators, including first author Stefan Siebert, used Drop-seq to obtain transcriptomes for 25,000 single cells from Hydra. "We just want to have a more complete catalogue of the cell types in Hydra and understand the genes that are expressed in those differentiated cell types, including the nervous system", explains Juliano as the motivation behind their study. In addition, Jack Cazet in Juliano's team performed ATAC-seq to identify putative regulatory sequences.

The cells analyzed with Drop-seq clustered into several cell types, including twelve types of neurons, four types of nematocytes, gland cells and germ cells, as well as multipotent interstitial stem cells. However, many cells were in an intermediate or transitional stage, and Juliano mentioned that certain cells can also transdifferentiate into others. Hence, Juliano is actually uncomfortable when talking about cell types. She points out that, "we should probably think about what do we mean when we say 'cell type', how do we really count the number of types of cells that are in a tissue or an organism. I think it is actually very challenging. The lines are very blurred." Instead, she and her colleagues prefer the term 'cell state'.

The single-cell sequencing approach provided Juliano and her collaborators with access to those cells that are transitioning between stem cells and differentiated cells. "You can't really capture those through bulk RNA sequencing," says Juliano. Hydra is particularly handy here, as cell transitions are "happening all the time, we only have to sequence the adult polyp and we can get a lot of information," says Juliano.
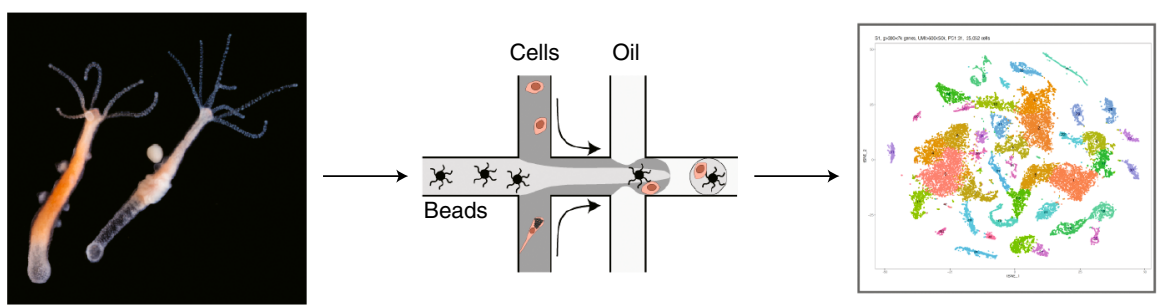

Hydra organisms are dissociated, prepared for Drop-seq, and the data are then clustered. Figure adapted from Siebert, S. et al. Science 365, 341 (2019).

Based on the Drop-seq dataset, the team could deduce lineage trajectories for all Hydra cells. Of note, for the computational aspect of the work on lineage trajectories, a collaboration with Jeffrey Farrell from Harvard University was crucial. Farrell, whom Juliano met at a developmental biology conference a few years ago, had developed an algorithm for finding cell trajectories based on following gene expression through the different stages of the zebrafish embryo. For the Hydra application, they had to modify the algorithm to account for the absence of prior knowledge of the temporal sequence in gene expression.

Importantly, although stem cells and their lineages are well studied in Hydra, the molecular underpinnings remained to be determined. "Now we can really better understand how these processes happen," says Juliano. She was particularly pleased that earlier work on cell trajectories from the 1980s and 1990s, which did not rely on the sophisticated tools available now, completely held up. Since Juliano and her colleagues generated ATAC-seq data in addition to the Drop-seq data, they could also look into the regulatory relationships of genes that they thought were involved in the differentiation processes.

With the modern tools, the team can go beyond this earlier work. Of particular interest to Juliano and her team is a previously unknown bipotential progenitor cell. They discovered that neurons and gland cells might share a common progenitor state that is different from the progenitors for nematocytes. They hypothesize that a population of the neuron-gland progenitors is located in the ectoderm and generates neurons there. Another population of these progenitor cells might cross over to the endoderm and gives rise to neurons or gland cells. In the future, Juliano and her colleagues plans to follow up on this observation.

In addition to the description of intermediate cell states, the single-cell sequencing approach also helped Juliano and her colleagues produce a molecular map of the nervous system. "We want to understand how the nervous system develops, how is it maintained in this homeostatic animal [and] how do you regenerate the nervous system," says Juliano. The molecular information obtained will allow them to manipulate the nervous system to address these questions.

Finally, Juliano also plans to manipulate signaling pathways or transcription factor expression and then to repeat the single-cell sequencing to see how these manipulations affect different lineage trajectories. "I think the future of developmental biology will go this way, where after doing some kind of perturbation, you don't just do bulk RNAseq or you don't just check a few genes, you actually do single-cell RNA-seq and find out exactly what has happened to everything," says Juliano.

Nina Vogt

Published online: 30 August 2019 https://doi.org/10.1038/s41592-019-0569-6

Research paper

Siebert, S. et al. Stem cell differentiation trajectories in Hydra resolved at single-cell resolution. Science 365, 341 (2019). 